\title{
Complementariedad de métodos y fuentes en el estudio histórico de familias indígenas (Belén, Altos de Arica, 1750-1820)
}

\section{Complementarity of methods and sources in the historical study of indigenous families (Belén, Altos de Arica, 1750-1820)}

\author{
Xochitl G. Inostroza Ponce ${ }^{1}$
}

\section{Resumen}

La reconstitución de familias del pueblo de Belén me ha permitido acceder a aspectos demográficos escasamente estudiados en poblaciones andinas, pero también a otros fenómenos cualitativos que permiten un análisis histórico de aspectos relativos al matrimonio y el parentesco. Contar con una gran cantidad de fuentes y archivos de distinta naturaleza ha desarrollado las potencialidades metodológicas, dando acceso a la comparación de resultados a partir de los distintos documentos, promoviendo una visión crítica del análisis individual de cada una de las fuentes, así como de los resultados de los diversos métodos. La complementariedad entre ellos facilita una comprensión global de los variados aspectos que intervienen en la conformación de las familias. En el caso de Belén, la integración de registros parroquiales, revisitas y un padrón, informes eclesiásticos y pliegos matrimoniales, me han llevado a distinguir fenómenos tales como la presencia de familias compuestas, dos posibles casos de poliginia, la tendencia masculina a la endogamia al interior del pueblo, las posibles preferencias que regulan los matrimonios -sobre todo en primeras nupcias-, así como a observar las dificultades demográficas y sociales que me llevan a hipotetizar respecto del rol que jugaron estos factores en contra de la familia extensa y a favor del parentesco ritual.

Palabras claves: reconstitución de familias, promedio de hijos, mortalidad, endogamia, Altos de Arica.

\begin{abstract}
The reconstitution of families of Belén gave me access not only to demographic aspects scarcely studied in Andean populations, but also to other qualitative phenomena, that allow a historical analysis related to marriage and kinship. The use of different kind of files and sources has strengthened the methodology development, which allows the comparison of different documents, fomenting a critical review of each of the sources as well as the results obtained using the various methods. Complementarity between them provides a comprehensive understanding of the various aspects involved in the formation of families. In the case of Belén, the integration of parish registers, revisitas and one padrón, church reports and marriage certificate have led me to distinguish phenomena such as the presence of compound families, two possible cases of polygyny, the male tendency towards endogamy within the village, possible preferences regulating marriages, especially concerning first marriage, as well as to observe demographic and social problems that lead me to hypothesize about the role those factors played in favor of ritual kinship and against extended family.
\end{abstract}

Keywords: reconstitution of families, average of children, mortality, inbreeding, Altos de Arica.

Recibido: 25 abril 2016. Aceptado: 26 julio 2017

1 Proyecto Fondecyt Postdoctoral 3180287 "Población, familia y vida cotidiana en espacios étnicos. Doctrina de Belén y sus fronteras (1750-1820)”. Centro de Estudios Culturales Latinoamericanos, Universidad de Chile. Email: xochitlinostroza@gmail.com 


\section{Introducción}

En el presente texto me interesa destacar los importantes alcances que el método de reconstitución de familias (Fleury y Henry, 1956; Henry, 1983) permite en el estudio histórico de las poblaciones (Robichaux, 1997, 2001; Amorin, 1995), ${ }^{2}$ lo que adquiere un valor aún más significativo si se trata de sujetos indígenas rurales, pues es el sector del que se encuentra menor cantidad de documentación en los archivos tradicionales. Pretendo además demostrar cómo la complementariedad de métodos y fuentes permiten explicar de mejor manera ciertos fenómenos demográficos y sociales, así como profundizar en las características de las familias que ha develado su reconstitución.

Debo partir por aclarar que utilizo el modelo de familia nuclear como categoría de análisis, como concepto-tipo, ${ }^{3}$ adoptado desde la demografía histórica y la historia de familia en América Latina, con la finalidad de promover un diálogo fructífero, interdisciplinario y que, por otra parte, sea lo suficientemente flexible como para respetar la diversidad de situaciones. Para la demografía histórica por ejemplo,

\section{[...] la familia se compone del matrimonio o del cónyuge superviviente $y$, eventualmente, de los hijos supervivientes; según esta defini- ción, un viudo o una viuda sin hijos forman una familia; los hijos considerados como miembros de una familia pueden ser, según el caso, los hijos de cualquier edad, los hijos su- pervivientes menores de cierta edad, los hijos solteros de cualquier edad que viven en el ho- gar paterno, los hijos menores de cierta edad que viven con sus padres (Henry, 1983, p. 31).}

Por razones históricas y de contexto, en mi investigación he agregado en esta caracterización a los grupos formados por mujeres, solteras o viudas, con hijos.

2 Para una discusión sobre la aplicación del método de reconstitución de familias en poblaciones americanas, remítase a Robichaux (2001).

3 "Como en toda ciencia generalizadora, es condición de la peculiaridad de sus abstracciones el que sus conceptos tengan que ser relativamente vacíos frente a la realidad concreta de lo histórico" (Weber, 2002, p. 16).
La reconstitución de familias del pueblo de Belén me ha permitido acceder a aspectos demográficos raramente estudiados en poblaciones andinas, centrados principalmente en la familia nuclear y el ciclo vital (Inostroza, 2014), ${ }^{4}$ pero también a otros fenómenos cualitativos que otorgan un análisis histórico a aspectos relativos al matrimonio y el parentesco (Inostroza, 2013a, 2016b). Por otra parte, las fichas de la reconstitución de familias pueden ser utilizadas por otros métodos para el estudio de las genealogías y las redes.

La investigación completa se ha enfocado en la Parroquia de Belén, compuesta por diez pueblos ubicados en la zona del valle interior y sierra de la provincia de Arica, cuya población era mayoritariamente indígena (Hidalgo, 1978; Hidalgo et al., 1988; Hidalgo et al., 2004; Inostroza, 2016a). La reconstitución de familias, en cambio, se realizó exclusivamente para el pueblo de Belén, debido a corresponder éste al pueblo capital de la doctrina y a que presenta características de una sociedad con un importante grado de cohesión social (Inostroza, 2016a). En comparación a localidades del Alto Perú (Klein, 1995), se trata de pueblos pequeños cuyo número no supera las 500 personas en los pueblos de mayor envergadura, incluso los más pequeños llegan a unas 40 personas, mientras que la mayoría apenas supera el centenar de habitantes.

El método consiste en reconstruir unidades familiares nucleares en "fichas de familias" que se completan a partir de los datos dispersos en los registros parroquiales. En este caso, ha sido aplicado a los registros del pueblo de Belén, cabecera de la doctrina que llevaba su nombre. ${ }^{5}$ Se identificaron 146 matrimonios de habitantes del pueblo entre 1774 y 1813 , de los cuales se obtuvo un $61 \%$ de fichas completas (89 familias), es decir, de núcleos familiares en los que se ha reconocido una fecha de inicio y una de término de la unión de la pareja (Figura 1), donde cada nuevo matrimonio representa una familia, incluso en casos de segundas nupcias. Con estas fichas es posible observar la fertilidad, el promedio de hijos por familia y la duración de los matrimonios, entre otros aspectos.

4 Sobre estas temáticas ver también Gil Montero (2004).

5 Archivo Nacional. Parroquia de Belén, 11 volúmenes. 


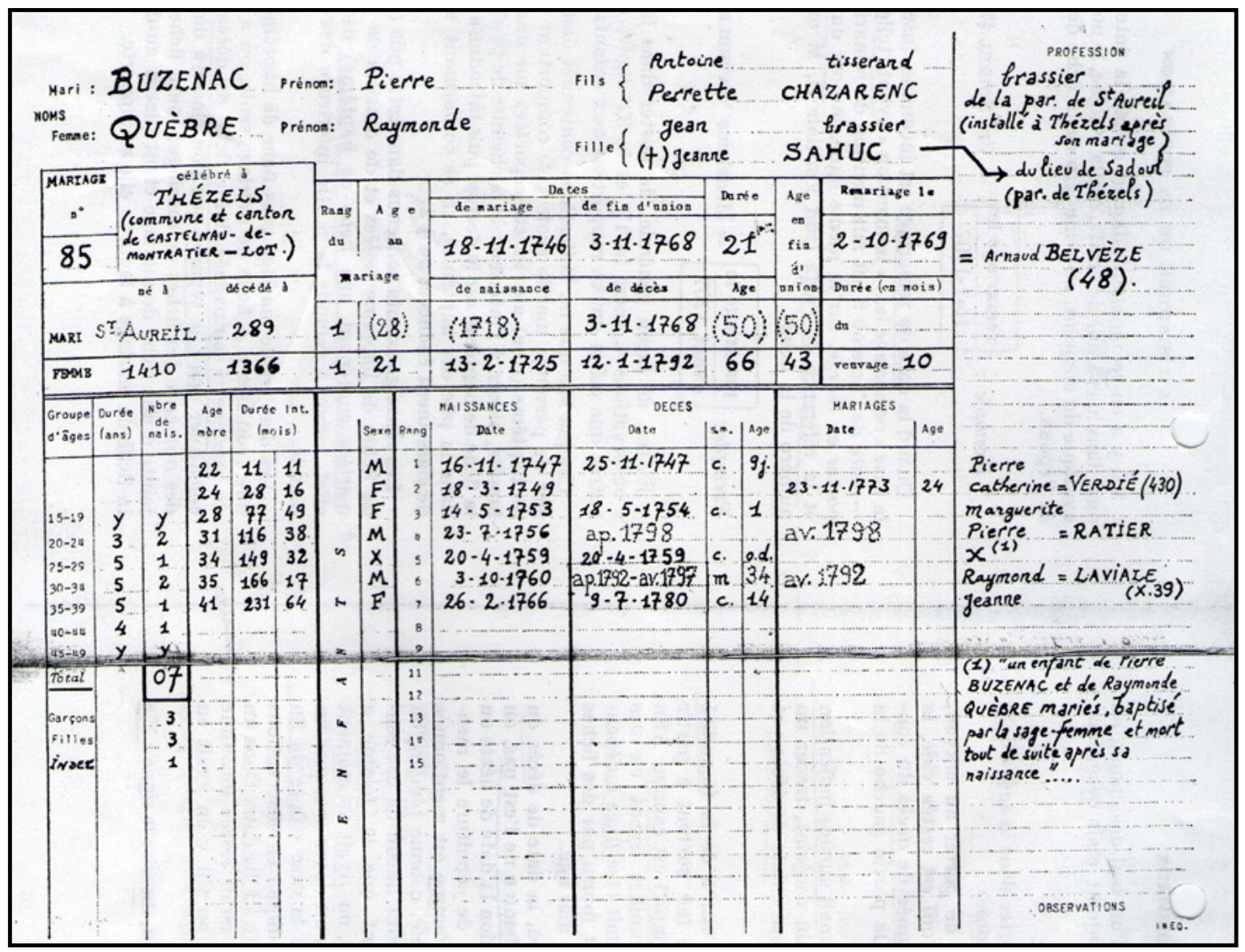

Figura 1. Ficha de familia de Fleury y Henry. ${ }^{6}$

Ahora bien, la aplicación de este método ha sido exitosa a mi juicio por tres factores principales. En primer lugar, debido a la buena calidad de las inscripciones parroquiales, la mayoría de las cuales se realizó con una dirección eficiente, la del padre Mariano Pacheco de Peñalosa, quien ofició como cura vicario durante gran parte del período que abarca este estudio. En segundo lugar, la existencia de registros de tipo censal en períodos colindantes a los registros parroquiales (Hidalgo, 1978; Hidalgo et al. 1988; Hidalgo et al., 2004), útiles para la identificación de "nacimientos recuperados" y "nacimientos perdidos" que contribuyen a completar las fichas de familia. Además, en el caso exclusivo de Belén, una de estas inscripciones, la Revisita de 1772/73, ha hecho posible la identificación de buena parte de

6 Fuente: Fleury y Henry, 1956, pp. 66-67. Esta ficha ha sido adaptada a una planilla Excel. las familias reconstituidas en uno de los dos ayllus en que se dividía el pueblo (Hidalgo et al., 2004). Finalmente un último elemento es la presencia de apellidos -mayoritariamente invariables- en ambos tipos de documentos, que además coinciden con otras fuentes eclesiásticas, permitiendo el reconocimiento de parte importante de la población que habitaba el pueblo de manera permanente.

\section{¿El pequeño tamaño de la familia?}

Como mencioné, la reconstitución de familias permite observar fenómenos demográficos relacionados con la fertilidad y el ciclo vital. En esta oportunidad me interesa destacar tres aspectos que se encuentran estrechamente relacionados: promedio de hijos por familia, edad al momento del matrimonio y duración del vínculo. En primer lugar, las 89 familias presentan un promedio de hijos bas- 
tante bajo, de 1,89 por familia. Ahora bien, este grupo de familias presenta características muy heterogéneas, pero al observar algunas de ellas se pueden comprender las razones que explican esta cifra. Una es la edad de los contrayentes. Las mujeres solteras se casaban en promedio a los 21 años (con una mediana de 20 ańos), mientras que las viudas se volvían a casar en promedio a los 33 años (con una mediana de 32 ańos). Los hombres solteros presentan un promedio y mediana de 23 años al momento de casarse, mientras que los viudos una edad promedio de 35 años (y mediana de 36) al contraer segundas nupcias. Pese a una tardía edad al matrimonio en solteros, se observa una edad bastante cercana en el matrimonio de viudos. Se debe advertir que estas edades no son otorgadas por el registro, sino que están calculadas a partir de la fecha de nacimiento.

Un segundo aspecto se relaciona con el anterior. Los matrimonios presentaban una corta duración, que en promedio alcanzaba los 9,95 años (y una mediana de 7,02), aunque la desviación estándar es alta $(9,33)$. En otras palabras, los matrimonios en Belén en muchos casos duraban poco, debido a la alta mortalidad de alguno de los cónyuges. Por lo tanto, una corta extensión de la vida marital explica, en gran parte, el bajo promedio de hijos (Inostroza, 2014).

Pero además, al fijar la atención en las diferencias entre las familias con mayor número de descendencia con respecto a las de menor envergadura, se vislumbra otro factor. En la Tabla 1 se observa la clasificación de las familias según el número de hijos, donde 29 de ellas no registraron ninguno $(33 \%)$. Seis de ellas corresponden a familias en que al menos uno de los cónyuges era viudo, mientras que en otros ocho casos, ambos lo eran. El factor viudez por lo tanto corresponde a 14 casos (16\% del total de familias). En ocho parejas se registró la muerte temprana de alguno de ellos (generalmente menos de dos años después del matrimonio), mientras que en cuatro ocasiones, uno era forastero o proveniente de otro pueblo de la doctrina. Solo en tres casos no se observan razones aparentes para la inexistencia de hijos, ya que se debe a parejas jóvenes, en primeras nupcias.
Tabla 1. Clasificación de las familias según el número de hijos. Belén, 1774-1813.

\begin{tabular}{|c|c|c|c|}
\hline $\mathbf{N}^{\circ}$ de hijos & $\begin{array}{c}\mathbf{N}^{\circ} \mathbf{d e} \\
\text { familias }\end{array}$ & $\%$ & $\begin{array}{c}\text { Total de } \\
\text { hijos }\end{array}$ \\
\hline 0 & 29 & 32,58 & 0 \\
\hline 1 & 21 & 23,60 & 21 \\
\hline 2 & 8 & 8,99 & 16 \\
\hline 3 & 9 & 10,11 & 27 \\
\hline 4 & 8 & 8,99 & 32 \\
\hline 5 & 3 & 3,37 & 15 \\
\hline 6 & 6 & 6,74 & 36 \\
\hline 7 & 1 & 1,12 & 7 \\
\hline 8 & 0 & 0,00 & 0 \\
\hline 9 & 3 & 3,37 & 27 \\
\hline 10 & 1 & 1,12 & 10 \\
\hline Total & 89 & 100 & 191 \\
\hline
\end{tabular}

La misma situación queda en evidencia en la Tabla 2 , donde se observa la cantidad de hijos registrados de los sujetos de autoridad, en relación a la secuencia matrimonial en que se encontraba, es decir, si correspondía a la primera, segunda, tercera y hasta cuarta unión. La mayoría de los primeros matrimonios registran varios hijos (de 5 a 11 registros), mientras que quienes se encontraban en segundas nupcias no registraron más de tres. En resumen, se puede afirmar que las familias compuestas por viudos tendían a inscribir menor cantidad de hijos.

Ahora bien, en cuanto al cálculo del promedio de hijos, el método de Henry recomienda realizar ciertos ajustes debido a la cantidad de niños que probablemente no fueron inscritos en los libros parroquiales, los que son denominados "nacimientos recuperados" y "nacimientos perdidos" (Fleury y Henry, 1956). En la Tabla 3, se observa que el promedio de hijos por familia -que se había establecido en 1,89 - asciende a 2,15 al incluir 23 nacimientos recuperados. $^{7}$

7 Los nacimientos recuperados corresponden a aquellos en que la mención a un hijo de la familia proviene de un registro de matrimonio, defunción o de un registro censal y no están en la inscripción bautismal. En los casos 
Tabla 2. Número de hijos de autoridades de Belén (1773-1833).

\begin{tabular}{|c|c|c|c|c|}
\hline $\begin{array}{l}Z \\
\text { Z } \\
\vdots \\
\vdots \\
\sigma \\
0\end{array}$ & ڤ્ఝ & $\overbrace{0}^{T}$ & $\begin{array}{l}\Xi_{0} \\
\text { E्: } \\
\text { : }\end{array}$ & 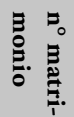 \\
\hline Tarque Jacinto & $\begin{array}{l}\text { Alcalde } \\
\text { interino }\end{array}$ & 1808 & 0 & $1^{\circ}$ \\
\hline Calle Fernando & Segunda & 1793 & 1 & $1^{\circ}$ \\
\hline Agnocuti Josef & Alcalde & 1790 & 2 & $1^{\circ}$ \\
\hline Aguilar Lino & Alcalde & 1833 & 5 & $1^{\circ}$ \\
\hline Cutipa Mariano & $\begin{array}{l}\text { Alcalde } \\
\text { Ordinario }\end{array}$ & 1816 & 5 & $1^{\circ}$ \\
\hline Marca Isidro & $\begin{array}{l}\text { Alcalde } \\
\text { Ordinario }\end{array}$ & 1809 & 5 & $1^{\circ}$ \\
\hline Ramos Ilario & Principal & 1773 & 5 & $1^{\circ}$ \\
\hline Agnocuti Justo & $\begin{array}{l}\text { Alcalde } \\
\text { Ordinario }\end{array}$ & 1810 & 6 & $1^{\circ}$ \\
\hline $\begin{array}{l}\text { Vasques } \\
\text { Gregorio }\end{array}$ & $\begin{array}{l}\text { Alcalde } \\
\text { Ordinario }\end{array}$ & 1794 & 6 & $1^{\circ}$ \\
\hline Alanoca Juan & $\begin{array}{l}\text { Alcalde } \\
\text { Mayor }\end{array}$ & 1797 & 7 & $1^{\circ}$ \\
\hline Cruz Alejandro & $\begin{array}{l}\text { Alcalde } \\
\text { Ordinario }\end{array}$ & 1792 & 7 & $1^{\circ}$ \\
\hline Maquera Asensio & Principal & 1773 & 8 & $1^{\circ}$ \\
\hline Marca Rafael & $\begin{array}{l}\text { Alcalde } \\
\text { Ordinario }\end{array}$ & 1803 & 9 & $1^{\circ}$ \\
\hline Larva Eduardo & $\begin{array}{l}\text { Alcalde } \\
\text { Mayor }\end{array}$ & 1800 & 10 & $1^{\circ}$ \\
\hline Bustos Florencio & $\begin{array}{l}\text { Alcalde } \\
\text { Ordinario }\end{array}$ & 1809 & 11 & $1^{\circ}$ \\
\hline Guanca Antonio & $\begin{array}{l}\text { Alcalde } \\
\text { Ordinario }\end{array}$ & 1796 & 3 & $2^{\circ}$ \\
\hline Guanca Eugenio & $\begin{array}{l}\text { Alcalde } \\
\text { Mayor }\end{array}$ & 1807 & 0 & $2^{\circ}$ \\
\hline $\begin{array}{l}\text { Larva Vicente/ } \\
\text { Santiago }\end{array}$ & $\begin{array}{l}\text { Alcalde } \\
\text { Mayor }\end{array}$ & 1803 & 0 & $2^{\circ}$ \\
\hline Marca Tomás & $\begin{array}{l}\text { Alcalde } \\
\text { Ordinario }\end{array}$ & 1789 & 2 & $2^{\circ}$ \\
\hline Nina Juan & Alcalde & 1813 & 0 & $2^{\circ}$ \\
\hline $\begin{array}{l}\text { Ocharan } \\
\text { Francisco }\end{array}$ & $\begin{array}{l}\text { Alcalde } \\
\text { Mayor }\end{array}$ & 1789 & 0 & $3^{\circ}$ \\
\hline Larva Jossef & $\begin{array}{l}\text { Alcalde } \\
\text { Mayor }\end{array}$ & 1785 & 1 & $4^{\circ}$ \\
\hline Maquera Martín & $\begin{array}{l}\text { Alcalde } \\
\text { Ordinario }\end{array}$ & 1793 & 2 & $4^{\circ}$ \\
\hline
\end{tabular}

Tabla 3. Promedio de hijos por familia más nacimientos recuperados (todas las familias).

\begin{tabular}{|c|c|c|c|c|c|}
\hline $\begin{array}{c}\mathbf{N}^{\circ} \text { de } \\
\text { familias }\end{array}$ & $\begin{array}{c}\mathbf{N}^{\circ} \text { de } \\
\text { hijos }\end{array}$ & P1 & Nr & $\begin{array}{c}\text { total } \\
\text { hijos }\end{array}$ & P2 \\
\hline 89 & 168 & 1,89 & 23 & 191 & 2,15 \\
\hline
\end{tabular}

Por otra parte debido a las características de las familias de Belén que ya he mencionado, propongo observar los resultados solo en las familias con hijos, excluyendo a las que no los registraron. En la Tabla 4 se representan las 60 familias que registraron al menos un hijo, los que suman una cantidad de 168 bautizados. El promedio de hijos en este caso asciende a 2,8, pero al agregar los nacimientos recuperados $(\mathrm{Nr})$, la cifra llega a los 3,18.

Tabla 4. Promedio de hijos por familia más nacimientos recuperados (familias con hijos).

\begin{tabular}{|c|c|c|c|c|c|}
\hline $\begin{array}{c}\mathbf{N}^{\circ} \text { de } \\
\text { familias } \\
\text { con } \\
\text { hijos }\end{array}$ & $\begin{array}{c}\mathbf{N}^{\circ} \text { de } \\
\text { hijos }\end{array}$ & $\mathbf{P 1}$ & $\mathbf{N r}$ & $\begin{array}{c}\text { total } \\
\text { hijos }\end{array}$ & $\mathbf{P 2}$ \\
\hline 60 & 168 & 2,80 & 23 & 191 & 3,18 \\
\hline
\end{tabular}

El método de Henry sugiere además agregar al total de niños registrados, la cifra calculada de nacimientos perdidos. Propone la siguiente fórmula: “[...] la relación entre el número de nacimientos perdidos y el número de nacimientos inscritos es igual a la relación análoga, calculada a partir de la fracción de los hijos de dichas familias que se hallan mencionados en un documento posterior y distinto del registro de bautismos o de nacimientos" (Henry, 1980, p. 123). ${ }^{8}$ La Tabla 5 muestra el cálculo de nacimientos perdidos en el pueblo de Belén según decenios. La mayor cifra en el decenio 1804-1813 corresponde al

en que solo se tenía la mención al año del nacimiento se procedió "como si el nacimiento se hubiera producido el 1 de enero del ańo supuesto" (Henry, 1983, p. 117).

8 Henry entrega un ejemplo que ayuda a comprender mejor la operación que se debe realizar en este caso: "Si, por ejemplo, en las familias surgidas de un casamiento de 1740-1769, tenemos 3 matrimonios de hijos cuyo nacimiento no ha sido registrado, y 30 que sí lo han sido, y que el número de nacimientos registrados de estas familias es de 90, el número de nacimientos perdidos es respecto a 90 como 3 es a 30, es decir, 9, décima parte de 90" (Henry, 1980, p. 123). 
Tabla 5. Evaluación de nacimientos perdidos. Matrimonios de Belén, 1774-1813.

\begin{tabular}{|l|c|c|c|c|}
\hline \multirow{2}{*}{$\begin{array}{c}\text { Decenio de } \\
\text { nacimiento }\end{array}$} & $\begin{array}{c}\text { Nacimientos registrados } \\
\mathbf{N r}\end{array}$ & \multicolumn{2}{|c|}{ Casamiento de hijos cuyo nacimiento está } & \multirow{2}{*}{$\begin{array}{c}\text { Evaluación nacimientos } \\
\text { perdidos }\end{array}$} \\
\cline { 3 - 4 } & 79 & $\begin{array}{c}\text { registrado } \\
\text { Cr }\end{array}$ & 2 & 5,45 \\
\hline $1774-1783$ & 40 & 29 & 0 & 0,00 \\
\hline $1784-1793$ & 28 & 9 & 2 & 7,00 \\
\hline $1794-1803$ & 18 & 8 & 4 & 24,00 \\
\hline $1804-1813$ & $\mathbf{1 6 5}$ & $\mathbf{4 9}$ & $\mathbf{8}$ & $\mathbf{2 6 , 9 4}$ \\
\hline Total & & & \\
\hline
\end{tabular}

período afectado al menos por tres acontecimientos trascendentales: la crisis de 1803 (Lima et al., 2016), que también se manifestó en el Alto Perú (Tandeter, 1991; Wachtel, 2001); el proceso de Juntas de Gobierno y abolición de los cacicazgos identificado por O'Phelan entre 1809 y 1814 (1997, 15), y la salida del cura vicario Mariano Pacheco de Peñalosa, quien estuvo encargado de organizar la doctrina y mantuvo los libros con notable celo. Ahora bien, se evidencia una disminución progresiva de los nacimientos registrados, lo que es coherente con la tendencia del registro de bautizos (Inostroza, 2016a).

La cifra total de nacimientos perdidos $(\mathrm{Np})$ calculados para el período completo $(26,94)$, puede ser agregada al total de niños de la Tabla 4 (191 hijos). De esta manera el promedio de hijos por familia asciende de 3,18 a 3,63 (Tabla 6).

Tabla 6. Promedio de hijos por familias más nacimientos recuperados y perdidos (familias con hijos).

\begin{tabular}{|c|c|c|c|c|c|}
\hline $\begin{array}{c}\mathbf{N}^{\circ} \text { de } \\
\text { familias }\end{array}$ & $\begin{array}{c}\mathbf{N}^{\circ} \text { de } \\
\text { hijos }\end{array}$ & P1 & Np & $\begin{array}{c}\text { total } \\
\text { hijos }\end{array}$ & P2 \\
\hline 60 & 191 & 3,18 & 26,94 & 218 & 3,63 \\
\hline
\end{tabular}

De esta manera, aplicando los ajustes propuestos por el método de reconstitución de familias y excluyendo a las familias sin hijos, se puede proponer que las familias de originarios en Belén (en primeras nupcias) tuvieron un promedio que oscila entre los 2 y 4 hijos, cifras mucho más probables que los 1,89 calculados inicialmente, y que, además, coincide con la información presentada en testamentos (Inostroza, 2013a).
Por otra parte, investigaciones demográficas del área andina de diversos contextos, muestran similitudes en cuanto al bajo promedio de hijos y la distribución de familias según su tamaño. Una de ellas es la Visita de Collaguas de 1591, en la que un total de 474 parejas registraron en promedio 1,33 hijos (Rénique y Trelles, 2012). La otra, es el análisis realizado por Fernando Ponce en la parroquia rural de Santa Marta (cercana a la ciudad de Arequipa), en la que utilizó un censo de 1813-1816 y los registros parroquiales correspondientes a las dos primeras décadas del siglo XIX. Ponce obtuvo un promedio de 2,95 hijos (Ponce, 1973, pp. 483, 486) (Tabla 7).

En los tres casos, las familias que no registraron hijos superan el 30\% del total de familias; las de Santa Marta incluso casi alcanzan al 50\%. ${ }^{9}$ Más aún, la mayoría de las familias de las tres localidades no superaron el hijo por familia. Pienso que pese a la diversidad de situaciones, los factores detectados en Belén (alta mortalidad, baja natalidad a partir del segundo matrimonio, corta duración de los matrimonios, etc.) pudieron estar presentes en los otros contextos, propiciando dichas similitudes. A pesar de las diferencias temporales, la comparación entre estas tres localidades es pertinente debido a que las tres incluyen espacios indígenas rurales del Arzobispado de Arequipa. Por otra parte, lo que me interesa destacar es la distribución en el número de hijos del registro y no su dimensión absoluta, pues lo que propongo es que condiciones similares debieron afectar esta proporción. En Collaguas, por ejemplo, los autores advierten la importante

9 El autor no entrega valores absolutos, solo porcentajes. 
Tabla 7. Número de hijos por familia. Belén (1774-1813), Collaguas (1591) y Santa Marta (1800-1820).

\begin{tabular}{|c|c|c|c|c|c|c|}
\hline \multirow{2}{*}{$N^{\circ}$ de hijos } & \multicolumn{2}{|c|}{ Belén } & \multicolumn{2}{|c|}{ Collaguas } & \multicolumn{2}{|c|}{ Santa Marta } \\
\hline & $\mathrm{N}^{\circ}$ de familias & $\%$ & $\mathrm{~N}^{\circ}$ de familias & $\%$ & $\mathrm{~N}^{\circ}$ de familias & $\%$ \\
\hline 0 & 29 & 33 & 152 & 32 & & 49 \\
\hline 1 & 21 & 24 & 130 & 27 & & 22 \\
\hline 2 & 8 & 9 & 104 & 22 & & 15 \\
\hline 3 & 10 & 11 & 64 & 14 & & 8 \\
\hline 4 & 7 & 8 & 19 & 4 & & 3 \\
\hline 5 & 3 & 3 & 3 & 1 & & 1 \\
\hline 6 & 6 & 7 & 2 & 0 & & 1 \\
\hline 7 & 1 & 1 & 0 & 0 & & 1 \\
\hline 8 & 0 & 0 & 0 & 0 & & 0 \\
\hline 9 & 3 & 3 & 0 & 0 & & 0 \\
\hline 10 & 1 & 1 & 0 & 0 & & 0 \\
\hline Total & 89 & 100 & 474 & 100 & & 100 \\
\hline
\end{tabular}

cantidad de parejas que no registraron hijos como uno de los factores que incide en el bajo promedio de hijos por familia:

Pensamos que este promedio es demasiado bajo y habría que replantear la forma en que se ha manejado esta información, pues es el caso que si nos fijamos un límite superior de edad para las parejas a considerar, reducimos la cantidad real y efectiva de hijos que cada una de esas parejas ha tenido. Veamos un par de ejemplos: en la visita encontramos una pareja de 53 años que no tiene registrado ningún hijo. Sin embargo, es perfectamente posible (y en más de un caso se ha podido demostrar) que esa pareja tenga uno o más hijos, de 20 años o más años Estos hijos, por el hecho de ser mayores, aparecen registrados en otra casa, si se han casado, o bajo el rubro de solteros (Rénique y Trelles, 2012, p. 125).
Las cifras de Santa Marta, por otra parte, son metodológicamente más cercanas a las de Belén, pues Ponce utilizó un método en el que combinó un registro censal con las actas de bautizo. En otro orden de ideas, es muy probable que las características de las familias campesinas sufrieran pocas alteraciones en el período colonial, salvo, claro está, en las épocas de crisis demográfica. Mellafe, por ejemplo, al estudiar el tamaño de la familia en la historia de América Latina entre 1562 y 1950, dio cuenta del tamaño de la familia nuclear en las localidades de Huánuco (1562), Songo, Challana y Chacapa (1568), Cusco (1690), Catamarca (1786), donde el tamańo de la familia fluctuó apenas de los 2,5 a los 3 integrantes (Mellafe, 2004 [1986], p. 248).

Volviendo a los factores, mencioné anteriormente otro aspecto que determina fuertemente el tamaño de la familia: la mortalidad de los adultos; fenómeno que ya se intuye al observar la corta duración de los matrimonios. Esta característica también puede ser analizada a partir de las fichas de familia: se 
calculó la edad de defunción en 93 casos de los hijos de las 98 familias completas, donde 70 sujetos fallecieron antes de cumplir los 15 años (75\%) y solo 23 lograron alcanzar edades adultas $(25 \%)$. El promedio de edad a la defunción fue de 28,96 años (con una mediana de 27, 44 y una desviación estándar de 9,75). Esta edad promedio es similar a la observada en otras localidades americanas en la época colonial, por ejemplo, en ciudad de México (Márquez y Civera, 1984, pp. 414-415).

La Tabla 8 muestra la distribución por edad y sexo de las defunciones de las parejas que conforman las familias completas (casados). Las mayores cifras se encuentran a los 40-49 ańos en el caso de los hombres y a los 30-34 años en el caso de las mujeres. Si se considera el promedio de edad de las personas fallecidas en dicho período como una especie de "esperanza de vida adulta”, ésta llega a los 40 ańos en ambos sexos. No se debe olvidar que esta expectativa corresponde a quienes lograron superar los 15 años de edad (un 25\% de la población).

Tabla 8. Distribución por edades y sexo de defunción de casados.

\begin{tabular}{|c|c|c|c|}
\hline Edad & Hombres & Mujeres & Total \\
\hline 15 a 19 & 0 & 3 & 3 \\
\hline 20 a 24 & 4 & 11 & 15 \\
\hline 25 a 29 & 9 & 3 & 12 \\
\hline 30 a 34 & 10 & 12 & 22 \\
\hline 35 a 39 & 3 & 8 & 11 \\
\hline 40 a 49 & 17 & 9 & 26 \\
\hline 50 a 59 & 6 & 8 & 14 \\
\hline 60 a 69 & 2 & 2 & 4 \\
\hline 70 a 79 & 1 & 7 & 8 \\
\hline 80 y más & 0 & 1 & 1 \\
\hline Desconocida & 37 & 25 & 62 \\
\hline Total & 89 & 89 & 178 \\
\hline
\end{tabular}

Como mencioné anteriormente, varias parejas estaban conformadas por al menos un viudo. Es así que la frecuencia de rematrimonios o matrimonios sucesivos es otro de los aspectos que afectó fuerte- mente el bajo promedio de hijos. La Tabla 9 muestra el estado matrimonial según sexo de las parejas reconstruidas. Las uniones entre solteros representan al 60\%, mientras que el matrimonio entre viudos o en que uno de los cónyuges lo es, alcanza al $40 \%$.

Tabla 9. Estado civil al momento de la unión.

\begin{tabular}{|c|c|c|c|}
\hline \multirow{2}{*}{ Mujeres } & \multicolumn{3}{|c|}{ Hombres } \\
\cline { 2 - 4 } & Solteros & Viudos & Total \\
\hline Solteras & 53 & 17 & 70 \\
\hline Viudas & 8 & 11 & 19 \\
\hline Total & 61 & 28 & 89 \\
\hline
\end{tabular}

\section{Familias, endogamia y relaciones de parentesco}

A partir de una lista de pagadores de diezmo de 1787, se reconstituyeron 69 familias nucleares del pueblo de Belén (Inostroza, 2016b). De las $69 \mathrm{fa}-$ milias, 45 corresponden a grupos nucleares formados por una pareja (65\%), 14 a familias encabezadas por mujeres solas (viudas o solteras, 20\%) y 10 a familias compuestas (15\%). Estas últimas integradas por sujetos provenientes de distintas unidades ante el fallecimiento de uno de sus progenitores y el posterior rematrimonio del padre o madre sobreviviente. Estas familias incluyeron en el grupo familiar no solo a los hijos de la pareja, sino que también a los hijos previos de ambos cónyuges. La existencia de familias compuestas muestra que la familia también era producto de una realidad social (Weismantel, 1998). El ejemplo que analizaré a continuación pone en evidencia además otra situación: un probable caso de poliginia.

En la Tabla 10 se revela la presencia de dos mujeres que son registradas como madres de algunos de los hijos "legítimos" de Josef Larva, pero que no contraen matrimonio con él, y que coexisten al mismo tiempo que otras mujeres "legítimas". De ninguna de ellas hay más información. Josef Larva procreó tres hijos con María Cutipa, que nacieron en forma alternada con los hijos de sus otras esposas. Con Juana Gaspar tuvo dos hijos, luego de lo cual su rastro desaparece. 
Tabla 10. Ejemplo de familia compuesta. Visión diacrónica.

\begin{tabular}{|c|c|c|c|}
\hline Nombre & $\begin{array}{l}\text { Parentesco con } \\
\text { J. Larva }\end{array}$ & $\begin{array}{l}\text { Edad [o fecha } \\
\text { de defunción] }\end{array}$ & Procedencia \\
\hline Larva Josef & & 45 & Aransaya \\
\hline Cruz Guanca Isabel & 1a esposa & $(+1774)$ & Aransaya \\
\hline Larva Francisco & Hijo $1^{\circ}$ matr. & $(+1784)$ & \\
\hline Cutipa Ma & Concubina 1? & ? & $?$ \\
\hline Diego & Hijo (concubina 1) & 23 & \\
\hline Gaspar Juana & Concubina 2? & $?$ & ? \\
\hline Larva Andrés & Hijo (concubina 2) & 22 & \\
\hline Larva Marcos & Hijo (concubina 2) & 20 & \\
\hline$M a$ & Hijo (concubina 1) & 15 & \\
\hline Larva Acencia & Hijo $\left(1^{\circ}\right)$ & 14 & \\
\hline Veles Bernarda & $2 a$ esposa & $(+1777)$ & Libilca/Umagata \\
\hline Larva Cruz Manuela & 3a esposa & $(+1784)$ & Aransaya \\
\hline Larva Martín & Hijo $\left(3^{\circ}\right)$ & 11 & \\
\hline Larva Antonio & Hijo $\left(3^{\circ}\right)$ & 9 & \\
\hline Larva Marta & Hijo $\left(3^{\circ}\right)$ & 8 & \\
\hline Vicente & Hijo $\left(3^{\circ}\right)$ & $(+1786)$ & \\
\hline Mollo Josefa & 4 a esposa & ? & Guallatiri \\
\hline Larva Melchora & Hijo $\left(4^{\circ}\right)$ & $* 1788$ & \\
\hline Pacsi Pedro & Hijo de Josefa Mollo & 18 (en Guallatiri) & \\
\hline Pacsi Manuel & Hijo de Josefa Mollo & $?$ & \\
\hline Pacsi Isabel & Hijo de Josefa Mollo & $(+1779)$ & \\
\hline Pacsi Blas & Hijo de Josefa Mollo & 6 & \\
\hline Cristina & Hijo (concubina 1) & ${ }^{*} 1791$ & \\
\hline
\end{tabular}


En la Tabla 11 se observan los personajes que integran la familia compuesta de Josef Larva en 1787: su cuarta mujer y los 10 hijos de distintas procedencias matrimoniales. Se observa al final de la tabla dos hijos que nacerían con posterioridad $\left(^{*}\right)$.

Tabla 11. Familia de Josef Larva en 1787.

\begin{tabular}{|c|c|c|c|}
\hline Nombre & $\begin{array}{c}\text { Parentesco } \\
\text { con } \\
\text { J. Larva }\end{array}$ & $\begin{array}{c}\text { Edad } \\
\text { [o fecha de } \\
\text { defunción] }\end{array}$ & Procedencia \\
\hline $\begin{array}{l}\text { Larva } \\
\text { Josef }\end{array}$ & & 45 & Aransaya \\
\hline $\begin{array}{l}\text { Cutipa } \\
\mathrm{Ma}\end{array}$ & Concubina 1? & ? & $?$ \\
\hline Diego & $\begin{array}{c}\text { Hijo } \\
\text { (concubina 1) }\end{array}$ & 23 & \\
\hline $\begin{array}{l}\text { Gaspar } \\
\text { Juana }\end{array}$ & Concubina 2? & ? & $?$ \\
\hline $\begin{array}{l}\text { Larva } \\
\text { Andrés }\end{array}$ & $\begin{array}{c}\text { Hijo } \\
\text { (concubina 2) }\end{array}$ & 22 & \\
\hline $\begin{array}{l}\text { Larva } \\
\text { Marcos }\end{array}$ & $\begin{array}{c}\text { Hijo } \\
\text { (concubina 2) }\end{array}$ & 20 & \\
\hline$M a$ & $\begin{array}{c}\text { Hijo } \\
\text { (concubina 1) }\end{array}$ & 15 & \\
\hline $\begin{array}{l}\text { Larva } \\
\text { Acencia }\end{array}$ & Hijo $\left(1^{\circ}\right)$ & 14 & \\
\hline $\begin{array}{l}\text { Larva } \\
\text { Martín }\end{array}$ & Hijo $\left(3^{\circ}\right)$ & 11 & \\
\hline $\begin{array}{l}\text { Larva } \\
\text { Antonio }\end{array}$ & Hijo $\left(3^{\circ}\right)$ & 9 & \\
\hline $\begin{array}{l}\text { Larva } \\
\text { Marta }\end{array}$ & Hijo $\left(3^{\circ}\right)$ & 8 & \\
\hline $\begin{array}{l}\text { Mollo } \\
\text { Josefa }\end{array}$ & 4 a esposa & $?$ & Guallatiri \\
\hline $\begin{array}{l}\text { Larva } \\
\text { Melchora }\end{array}$ & Hijo $\left(4^{\circ}\right)$ & 1788 & \\
\hline $\begin{array}{l}\text { Pacsi } \\
\text { Manuel }\end{array}$ & $\begin{array}{c}\text { Hijo de Josefa } \\
\text { Mollo }\end{array}$ & ? & \\
\hline Pacsi Blas & $\begin{array}{c}\text { Hijo de Josefa } \\
\text { Mollo }\end{array}$ & 6 & \\
\hline Cristina & $\begin{array}{c}\text { Hijo } \\
\text { (concubina 1) }\end{array}$ & *1791 & \\
\hline
\end{tabular}

Josef Larva fue alcalde mayor en 1793. El apellido Larva corresponde a una de las familias más antiguas originarias de Belén, y una de las más numerosas. Aparte de este, solo he identificado otro posible caso de bigamia, también de un sujeto principal, Francisco Ocharan, secretario del cabildo indígena (Inostroza, 2013a). Sin embargo, en ambos casos las evidencias son difusas, lo que puede responder al intento de ocultar situaciones de esta índole.

A partir de la lista de 1787, además, se pudo identificar la procedencia de ambos cónyuges en $40 \mathrm{ca}$ sos (Inostroza, 2016b), donde el 62\% de las parejas (25 casos) se casaron al interior del pueblo (ayllus Mancasaya y Aransaya). En cuanto al matrimonio de personas de distintos ayllus, las diferencias fueron poco significativas. Los hombres de Mancasaya muestran una leve tendencia al matrimonio con mujeres del mismo ayllu (15\%, correspondiente a seis casos), mientras que los de Aransaya casi no reflejan predilección (17\% en ambos casos, correspondiente a siete casos). Sin embargo, las 40 parejas incluyen a solteros y viudos, es decir, sujetos casados en segundas o terceras nupcias.

Diferente situación se presenta en la procedencia de los cónyuges de 38 parejas en primeras nupcias, provenientes de las fichas completas de la reconstitución de familias (1774-1813). En este caso la endogamia del pueblo es alta (97\%) debido a la discriminación de los registros de matrimonios que pertenecían al pueblo de Belén. Pese a ello, la misma situación queda manifiesta en los registros matrimoniales de la doctrina completa. ${ }^{10}$ A diferencia de la primera situación, en este caso el ayllu Mancasaya no mostró preferencias al matrimonio entre los dos ayllus $(28,9 \%$ correspondiente a 11 casos). Sin embargo, llama la atención el mayor porcentaje de los matrimonios realizados entre hombres provenientes del ayllu Aransaya con mujeres provenientes de Mancasaya (31,6\% correspondiente a 12 casos). Las cifras que se obtienen en ambas tablas, hacen probable la preferencia de uniones con sujetos provenientes de Mancasaya, lo que seguramente responde

10 La mayor tendencia de la endogamia al interior del pueblo se observa en otras localidades vecinas. Rivière lo observa en Sabaya en cifras comparativas que van desde el siglo XVIII al siglo XX (1982, pp. 243-247). Véase también Platt, 1980, entre otros. 
a una situación estratégica en el asentamiento de Belén (Inostroza, 2016b). Como se observa en estas dos últimas tablas, la reconstitución de familias ha posibilitado identificar de manera más clara un fenómeno particular: es en el matrimonio en primeras nupcias donde entran en juego mayoritariamente las preferencias matrimoniales.

Finalmente, hay otros dos métodos que se ven beneficiados por una reconstitución de familias. En primer lugar, la construcción de genealogías, que evidentemente requiere el rescate de todos los antecedentes que correspondan al grupo de descendencia que se pretende observar. En este sentido, el método francés facilita el trabajo genealógico, al organizar cada uno de los registros en fichas de familias.

En este sentido, el análisis de genealogías entrega mayor información respecto de la procedencia de los cónyuges. Muestra que eran los hombres quienes generalmente contraían matrimonios endogámicos a partir de un sistema que era principalmente patrilocal. Las mujeres en muchas ocasiones se casaban fuera del ayllu o lo hacían con sujetos de otros pueblos. Sin embargo, ante la ausencia de descendientes varones, las mujeres también se casaban con sujetos del mismo ayllu o del mismo pueblo (Inostroza, 2016b). Para ejemplificar mencionaré una situación.

Elegí la genealogía de Guillermo Maquera ${ }^{11}$ ya que dos de sus hijos ocuparon cargos de autoridad. El primer aspecto que destaca, es el hecho de encontrarse solo los registros de matrimonio de sus tres hijos varones. En cambio, de las tres hijas inscritas en su familia, en 1750 , no hay otros antecedentes en los libros parroquiales. Los tres hijos se casaron en primeras nupcias con mujeres de Belén; dos de ellas tienen procedencia del mismo ayllu que sus maridos. El hijo varón mayor, Asencio Maquera, fue principal en 1773 y alcalde mayor en $1780^{12}$ ( Figura 2). Él y su esposa registraron ocho hijos. El mayor, Felipe, se casó en dos oportunidades. Las dos esposas que tuvo provenían de familias de Mancasaya. No se pudo determinar el ayllu de procedencia de las esposas de sus dos hijos, pero sí se sabe que la

11 Registrado como Guillermo Maqueso en la Revisita de 1750 (Hidalgo, 1978, p. 105).

12 Archivo Nacional Parroquia de Belén vol. III, f. 122v esposa de su nieto Jerónimo también pertenecía a una familia proveniente del mismo ayllu. En cambio, una de las hijas de Asencio (la única que tiene registro de matrimonio) se casó con un sujeto de Aransaya: Simón Larva. En resumen, es muy probable que todas las mujeres casadas con descendientes de Asencio Maquera provinieran de Mancasaya. En el caso de Martín Maquera, segundo hijo de Guillermo Maquera, ocurre una situación diferente ( $\mathrm{Fi}$ guras 3, 4 y 5): su primera esposa era de su mismo ayllu, pero la segunda, de Aransaya y la tercera era forastera, de Curaguara. ${ }^{13}$ Sus dos hijos casados contrajeron matrimonio con sujetos foráneos al pueblo: Agustina, con Blas Pacsi y Leandro, con Marcelina Loaiza, de Choquelimpe. Cuatro de los descendientes de Asencio continuaban en Belén en 1813: su hija María y sus nietos Simona, Ramón ${ }^{14}$ y Felipe (Hidalgo et al., 1988, pp. 49-50). De los otros dos hermanos, solo quedaba Agustina, hija de Martín, casada con forastero. En síntesis, se observa en las alianzas matrimoniales la tendencia del hijo varón mayor a casarse al interior de su mismo ayllu, mientras que el matrimonio de los demás hermanos parece ser más flexible. Lo mismo se observa en segundas nupcias, incluso del primer hijo de la familia.

Por otra parte, este análisis puede ser revelador en un aspecto acerca de las fuentes de información: de los 11 descendientes varones de Guillermo Maquera se encuentra el registro de ocho matrimonios. En cambio de las 18 mujeres solo hay dos inscripciones. Ambas continúan en el pueblo hacia 1813. Mi hipótesis es que otras mujeres de la familia se casaron con sujetos de doctrinas vecinas, por lo que sus registros se encontrarían en las iglesias a donde llegaron a residir. Quizás esto pueda explicar la mayor frecuencia de matrimonio de los hombres en los libros parroquiales del que dan cuenta las genealogías que he analizado.

13 Pero Curaguara tampoco era cualquier pueblo. Era uno de los principales asentamiento carangas. La relación entre ambas zonas se discutirá en otra oportunidad. Al respecto ver: Hidalgo 1986, Chacama 2009, Horta 2011, entre otros.

14 Ramón fue registrado en 1813 como Pedro Maquera. Debió ocurrir un error al escribir los nombres de la pareja, pues su mujer, registrada como Petrona Alanoca en el registro de matrimonios, aparece en el padrón con el nombre de su marido, Ramona, y viceversa. 
Debido a la alta mortalidad adulta y la frecuencia de segundas nupcias, la conformación de familias compuestas debió ser muy recurrente. Ejemplo de ello sería la familia integrada por la viuda de Francisco Ocharan y los hijos de su consorte fallecido (Inostroza, 2013a). Estas situaciones me llevan a pensar en el impacto que debió causar en el entramado de las relaciones de parentesco, donde terminaban confluyendo varias familias, lo que me permite proponer dos hipótesis relacionadas. En primer lugar, que la alta mortalidad de los adultos y las altas frecuencias de rematrimonios generaron confusión entre las relaciones de parentesco. El pliego matrimonial de Tomás Marca -indio principal, alcalde y mayordomo del pueblo de Belén- muestra un ejemplo. Se da cuenta del parentesco por afinidad que tenía en segundo y tercer grado con Rosa Larva. ${ }^{15}$ Según la información matrimonial, ni los contrayentes ni los alcaldes del pueblo tenían clara la red parental que los unía, sin embargo, el nuevo doctrinero advirtió el parentesco al consultar a las ancianas del pueblo:

\section{[...] vine a descubrir que Diego Larba marido de Francisca Samorano procreo a Florentino y Ana Larba, aquél a Lucía madre de Rosa, y esta a Francisca consorte de Thomas, y se de- duce que Florentino, y Ana fueron hermanos, Lucia y Francisca primas hermanas, y por con- siguiente viene a resultar la mencionada Rosa sobrina segunda de Francisca Guanca muger de Marca, y en el mismo grado afines deste $[\ldots]{ }^{16}$}

Así, el proceso de reconstruir las familias del pueblo de Belén me ha llevado a proponer que muchos de los matrimonios fueron acordados, sobre todo aquellos que "tendrían por finalidad integrar a los sujetos 'desprotegidos' en núcleos familiares que les dieran sustento" (Inostroza, 2013b). Eso queda evidente en la información matrimonial de Tomás Marca. A pesar del parentesco, el cura Lorenso Pacheco de Peñalosa solicitó al Obispo la dispensa necesaria para el casamiento, apelando "... a la miserable condicion de estos naturales, y en especial a la orfandad de la contraiente que se alla con hun hermano menor y

15 Este y otros casos se desarrollan en Inostroza, 2013b.

16 Archivo Arzobispal de Arequipa. Arica-Belén, 16941856. Pliego matrimonial de Tomás Marca, 1802. hun sobrino guerfanos a su cargo que necesariamente exijen de ausilios y educación". ${ }^{17}$ En el caso de Francisco Ocharan, se observa cómo una mujer viuda, probablemente sin ningún familiar vivo, fue integrada a un nuevo núcleo, que la protegería incluso después de la muerte del marido con el que se instituyó el nuevo nexo (Inostroza, 2013a).

Por otra parte -y ésta sería la segunda hipótesis-, la misma situación (la desaparición de muchos miembros de la familia extensa) debió provocar la confusión de las redes de parentesco sanguíneo que propiciaron el éxito del establecimiento de relaciones de compadrazgo, como una manera de solucionar la necesidad de redes de reciprocidad y ayuda (Alberti y Mayer, 1974; Del Castillo, 1989). En cuanto a este tema, la reconstrucción de familias es útil en el análisis de redes sociales, ya que permite identificar las redes de cada individuo en particular, superando las dificultades que se enfrentan al establecer nexos a sujetos que tienen un mismo nombre (Inostroza, 2016a).

\section{Consideraciones finales}

La reconstitución de familias realizada a partir de los registros de bautizo, matrimonio y defunción del pueblo de Belén, y su triangulación con las revisitas de 1750, 1773 y el padrón de 1813, ha facilitado el acceso a aspectos demográficos históricos escasamente estudiados en sociedades andinas, relacionados con la familia y el ciclo vital. En primer lugar, permite corregir el promedio de hijos que se obtiene a partir del análisis de registros parroquiales, mostrando una cifra que oscila entre los 2 y 4 hijos por familia, aunque coincidiendo con una situación observada en localidades diversas en los Andes: el pequeño tamaño de la familia nuclear. Además, permite explicar las principales causas de este fenómeno, entre las que se encuentran: mortalidad, estado al matrimonio y origen. Pero además, el método contribuye en la observación de fenómenos cualitativos. A partir de la lista de pagadores de diezmos de 1787 y de la Revisita de 1773, se pudo dar cuenta de la conformación de familias compuestas, y de dos posibles casos de poliginia. Además se

17 Archivo Arzobispal de Arequipa. Arica-Belén, 16941856. Pliego matrimonial de Tomás Marca, 1802. 
pudo constatar el origen de 80 cónyuges, que muestra una tendencia a contraer matrimonios endogámicos a nivel de ayllu, mientras que las familias de la reconstitución completa (1774-1813) reflejan la preferencia al matrimonio con sujetos provenientes del ayllu Mancasaya. El análisis de genealogías, por otra parte, confirma esta situación, mostrando la predilección de los primeros matrimonios a contraer nupcias en Mancasaya, pero dando cuenta de la flexibilidad desde la segunda unión. Queda en evidencia que las características -tanto cuantitativas como cualitativas- de las familias conformadas por sujetos en primeras nupcias varían en las familias que ellos mismos conformaron posteriormente. Este elemento me parece central para ser tomado en cuenta por futuras investigaciones, ya que diversos fenómenos como el número de hijos y los porcentajes de endogamia varían notablemente entre primeras y segundas nupcias.

Propongo, además, que la alta mortalidad provocaba la rápida desintegración del grupo nuclear de procedencia, dando pie a nuevas configuraciones familiares y generando invisibilización y confusión entre las relaciones del parentesco y propiciando, seguramente, el fortalecimiento de las relaciones de compadrazgo. En este sentido, quizás estas características demográficas logren explicar la gran importancia que adquirió en los Andes el establecimiento del parentesco ritual.

Varios de los aspectos aquí abordados requieren de un análisis profundo de los diferentes aspectos contextuales que permiten explicarlos, lo que escapa de los objetivos del presente artículo. Mi intención ha sido relevar la importancia de la complementariedad de métodos y fuentes en el estudio histórico de la población y la familia, y los importantes alcances del método de reconstitución de familia, que me ha permitido observar de manera más clara factores $y$ variables que explican las características de las diversas familias indígenas que habitaron el pueblo de Belén, en las postrimerías del período colonial.

\section{Agradecimientos}

Agradezco a los profesores Jorge Hidalgo y Herbert Klein por su guía en mi investigación doctoral. A
David Robichaux y Juan Pablo Ferreiro por las apreciadas conversaciones sobre las familias indígenas. A los evaluadores anónimos por sus valiosos comentarios.

\section{Referencias citadas}

Alberti, G., y Mayer, E. (Comps.). (1974). Reciprocidad e intercambio en los Andes peruanos. Lima: Instituto de Estudios Peruanos.

Amorim, M. (1995). Informatizaçao normalizada de arquivos. Reconstiutiçao de paróquias e historia das populaçoes. Boletín de la ADEH XIII(2), 141-150.

Archivo Arzobispal de Arequipa. Arica-Belén, 1694-1856. Pliego matrimonial de Tomás Marca, 1802.

Archivo Nacional. Parroquia de Belén. 11 volúmenes.

Chacama, J. (2009). Imágenes y palabras, dos textos para un discurso: la prédica pastoral en los Andes coloniales, Doctrina de Codpa (Altos de Arica), siglo XVIII. Diálogo andino, 33, 7-27. http://www.redalyc.org/articulo. oa?id=371336243002

Del Castillo, A. M. (1989). Simbolismo y poder: un estudio antropológico sobre compadrazgo y priostazgo en una comunidad andina. Barcelona: Anthropos Editorial.

Fleury, M., y Henry, L. (1956). Des registres paroissiaux à L'Histoire de la population. Manuel de dépouillement et d'exploitation de l'état civil ancien. Paris: Institut National D’Études Démographiques.

Gil Montero, R. (2004). Caravaneros y trashumantes en los Andes meridionales. Población y familia indigena en la puna de Jujuy, 1770-1870. Lima: IEP.

Henry, L. (1983). Manual de demografía histórica. Barcelona: Crítica.

Hidalgo, J. (1978). Revisita a los altos de Arica efectuada por el oficial real don Joaquin de Cárdenas 1750. Iquique: Universidad del Norte.

Hidalgo, J. (1986). Indian Society in Arica, Tarapaca and Atacama, 1750-1793, and its response to the rebelion of Tupac Amaru. Thesis Ph. D. University of London. 
Hidalgo, J., Arévalo, P., Marsilli, M., y Santoro, C. (1988). Padrón de la Doctrina de Belén en 1813: un caso de complementariedad tardía. Arica. Arica: Universidad de Tarapacá, Facultad de Estudios Andinos. Departamento de Antropología, Geografía e Historia.

Hidalgo, J., Castro, N., y González, S. (2004). La Revisita de Codpa (Altos de Arica) de 1772-73 efectuada por el corregidor Demetrio Egan. Chungara. Revista de Antropologia Chilena, 36(1), 103-112. http://www.scielo.cl/ scielo.php?pid=S0717-73562004000100005\&script $=$ sci arttext\&tlng=pt

Horta, H. (2011). El gorro troncocónico o Chucu y la presencia de población altiplánica en el norte de Chile durante el Período Tardío (CA. 1.470-1.536 DC). Chungara. Revista de Antropología Chilena, 43 (Especial), 551-580. http://www.scielo.cl/scielo.php?pid=S071773562011000300012\&script=sci_arttext $\&$ tlng=e

Inostroza, X. (2013a). Dimensiones del liderazgo étnico en el pueblo de Belén: Francisco Ocharán, secretario del cabildo indígena. Altos de Arica (1750-1813). Estudios Atacameños. Arqueología y Antropología Surandinas, 46, 109-126. http://www.scielo.cl/scielo.php?pid=S0718 $10432013000200007 \&$ script=sci_arttext

Inostroza, X. (2013b). Políticas matrimoniales y prácticas indígenas. Doctrina de Belén, Altos de Arica (17631823). Revista Allpanchis, XLIV(81-82), 245-278.

Inostroza, X. (2014). Matrimonio y familia en sociedades andinas: propuestas desde la reconstitución de familias de Santiago de Tacrama o Belén. Virreinato del Perú (1763-1820). Historia, 47(1), 65-90. http://www.scielo.cl/ scielo.php?pid $=$ S0717-71942014000100003\&script $=$ sci_ arttext\&tlng=en

Inostroza, X. (2016a). Parroquia de Belén: Población, familia y comunidad en una doctrina de indios. Altos de Arica 1763-1820. Tesis para optar al grado de Doctora en Historia, mención Etnohistoria, Facultad de Filosofía y Humanidades, Universidad de Chile.

Inostroza, X. (2016b) Familias y ayllus: alianzas, riqueza y prestigio. Belén 1787 (Altos de Arica). Surandio Monográfico, (1), 6-23. http://revistascientificas.filo.uba.ar/ index.php/surandino/article/view/3502
Klein, H. (1995). Haciendas y Ayllus en Bolivia: la región de La Paz, siglos XVIII y XIX. Lima: Instituto de Estudios Peruanos.

Lima, C., Santoro, C., y Latorre, C. (2016). Coupled Socio-Environmental Changes Triggered Indigenous Aymara Depopulation of the Semiarid Andes of Tarapacá-Chile during the Late 19th-20th Centuries. PLoS ONE, 11(8), e0160580. https://doi.org/10.1371/journal. pone. 0160580

Márquez, L., y Civera, M. (1984). Paleodemografía de una muestra de población del periodo colonial mexicano. Estudios de antropología biológica, 3(3), 405-417.

Mellafe, R. (1980). Tamaño de la familia en la historia de Latinoamérica 1562-1950. En Mellafe, R. Historia social de Chile y América (pp. 231-250). Santiago: Editorial Universitaria, 2004.

O'Phelan, S. (1997). Kuracas sin sucesiones. Del cacique al alcalde de indios. Perú y Bolivia 1750-1835. Cusco: Centro de Estudios Andinos Bartolomé de Las Casas.

Plat, T. (1980). "Espejos y maíz. El concepto de Yanantin entre los macha de Bolivia. En Mayer, E., y Bolton, R. Parentesco y matrimonio en los Andes (pp. 139-182). Lima: Pontificia Universidad Católica del Perú, Fondo Editorial.

Ponce, F. (1978). Población y familia en una zona rural de Arequipa pre-republicana. En Miró, F., Pease F., y Sobrevilla D. Historia, problema y promesa (pp. 473-489). Lima: Pontificia Universidad Católica del Perú, Fondo Editorial.

Rénique, J. L., y Trelles, E. (2012). Aproximaciones demográficas, Yanque-Collaguas, 1591. En Robinson, D. (Ed.). Collaguas I. Visitas de Yanque-Collaguas, 1591 y documentos asociados (pp. 115-130). Lima: Pontificia Universidad Católica del Perú, Fondo Editorial.

Rivière, G. (1982). Sabaya: structures socio-économiques et representations symboliques dans le Carangas Bolivie. Tesis doctoral. París, École des Hautes Études en Sciences Sociales.

Robichaux, D. (1997). La reconstitution des familles en Amérique Latine. En Poussou, J.-P., y Robin-Romero, I. Histoires des familles, de la démographie et des compor- 
tements en hommage à Jean-Pierre Bardet (pp. 259-277). Paris: Presses Paris Sorbonne.

Robichaux, D. (2001). Uso del método de la reconstitución de familias en las poblaciones indígenas. Papeles de población, 28, 99-129.

Tandeter, E. (1991). Crisis in Upper Perú. Hispanic American Historical Review 71(1), 35-71. http://www.jstor.org/ stable/2516422?seq=1\#page_scan_tab_contents
Wachtel, N. (2001). El regreso de los antepasados. Los indios urus de Bolivia, del siglo XX al XVI. Ensayo de historia regresiva. México: FCE.

Weber, M. (2002). Economía y sociedad. México: FCE.

Weismantel, M. (1998). Viñachina: Hacer guaguas en Zumbagua, Ecuador. En Arnold, D. (Comp.). Parentesco y género en los Andes II. Gente de carne y hueso. Las tramas de parentesco en los Andes (pp. 83-96). La Paz: CLASE/ILCA.

\section{Anexos}

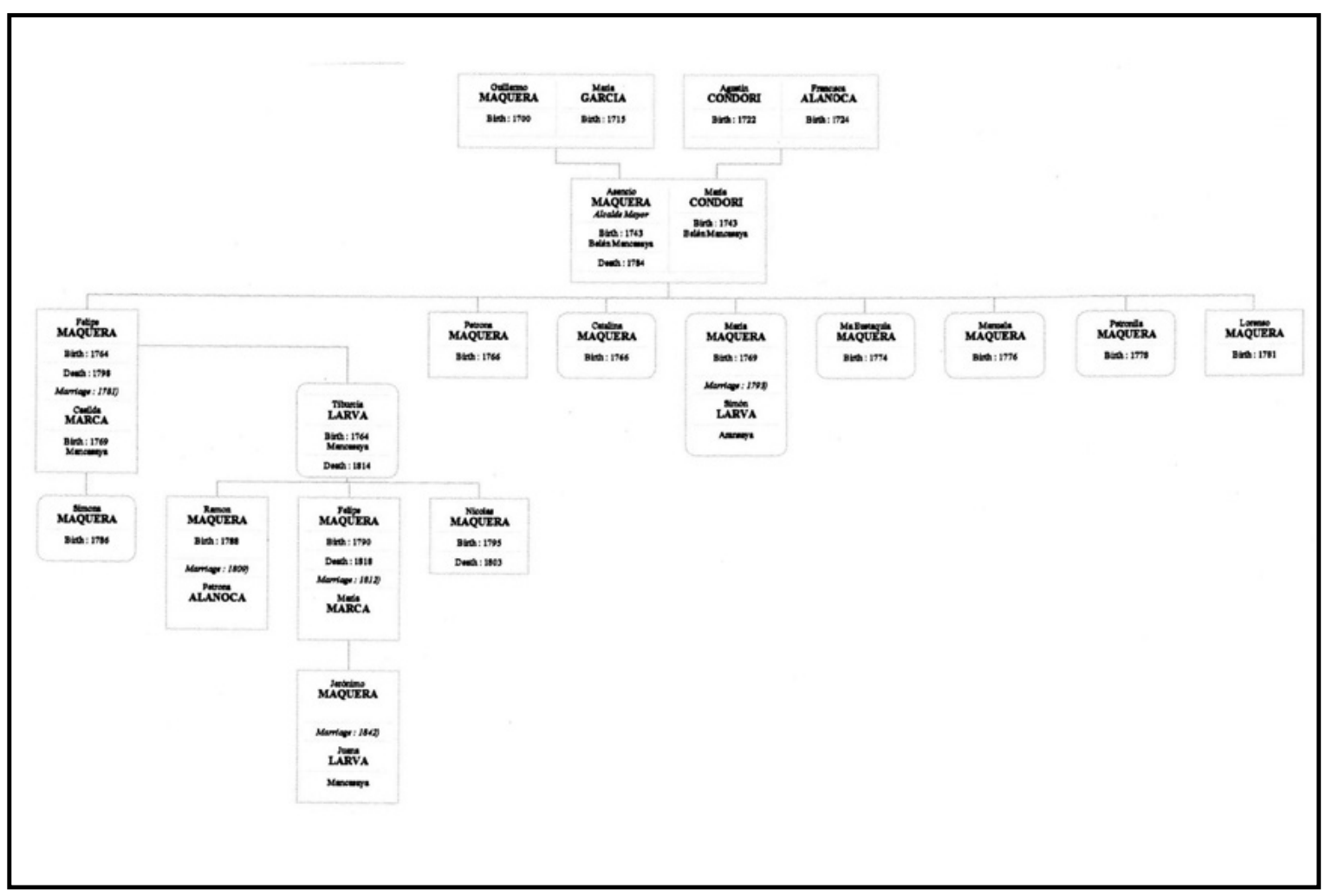

Figura 2. Genealogía de Asencio Maquera. 


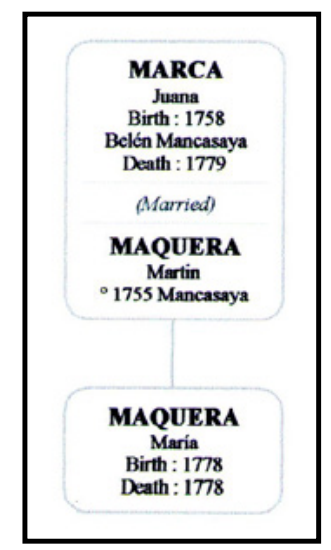

Figura 3. Genealogía de Martín Maquera y María Marca (1776-1779).

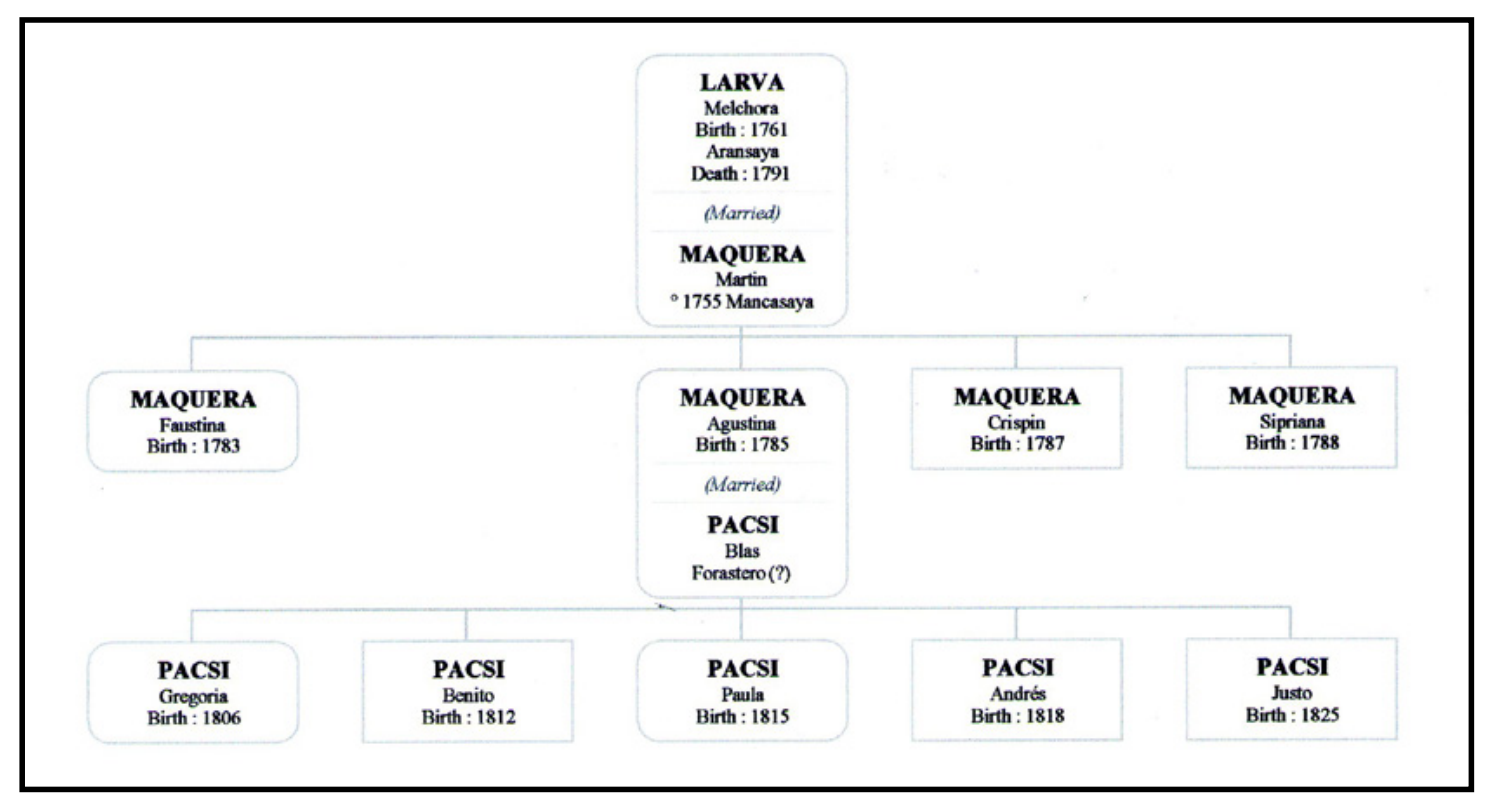

Figura 4. Genealogía de Martín Maquera y Melchora Larva (1780-1791). 


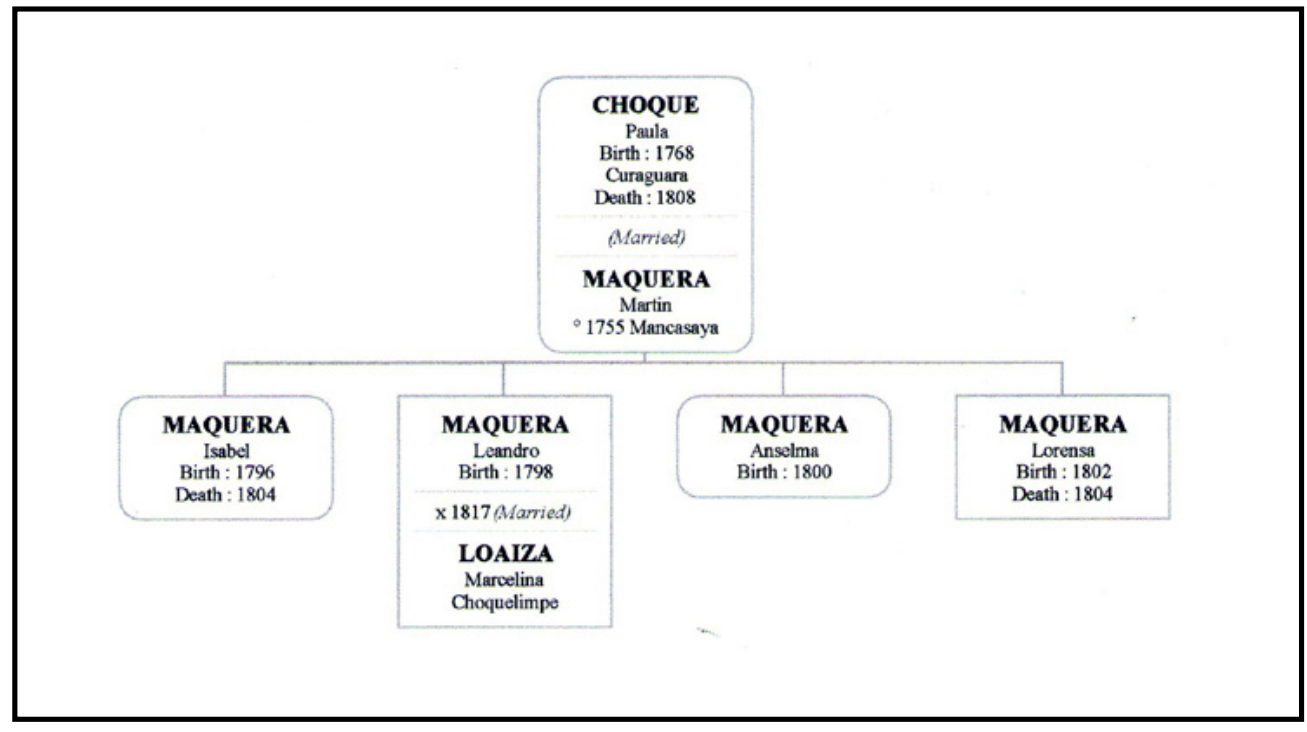

Figura 5. Genealogía de Martín Maquera y Paula Choque $(1792-1807(?))$. 